\title{
Control of Grip Force When Tilting Objects: Effect of Curvature of Grasped Surfaces and Applied Tangential Torque
}

\author{
Antony W. Goodwin, ${ }^{2}$ Per Jenmalm, ${ }^{1}$ and Roland S. Johansson ${ }^{1}$ \\ ${ }^{1}$ Department of Physiology, Umeå University, S-901 87 Umeå, Sweden, and 2Department of Anatomy and Cell Biology, \\ University of Melbourne, Parkville, Victoria 3052, Australia
}

\begin{abstract}
When we manipulate objects in everyday tasks, there are variations in the shape of the grasped surfaces, and the loads that potentially destabilize the grasp include time-varying linear forces and torques tangential to the grasped surfaces. Previous studies of the control of fingertip forces for grasp stability have dealt principally with flat grip surfaces and linear force loads. Here, we studied the regulation of grip force with changes in curvature of grasped surfaces and changes in tangential torque applied by the index finger and thumb when humans lifted an object and rotated it about the horizontal grip axis through an angle of $65^{\circ}$. The curvatures of the matched pair of spherical surfaces varied from $-50 \mathrm{~m}^{-1}$ (concave with radius $20 \mathrm{~mm}$ ) to $200 \mathrm{~m}^{-1}$ (convex with radius $5 \mathrm{~mm}$ ). The applied tangential torque at the orientation of $65^{\circ}$ was varied sixfold. Regardless of the values of curvature and end torque, grip force and
\end{abstract}

tangential torque were coordinated, increasing in parallel throughout the tilt with an approximately linear relationship; the slope of the line increased progressively with increasing surface curvature. This parametric scaling of grip force was directly related to the minimum grip force required to prevent rotational slip, resulting in an adequate safety margin against slip in all cases. We conclude that surface curvature parametrically influences grip force regulation when the digits are exposed to torsional loads. Furthermore, the sensorimotor programs that control the grip force apparently predict the effect of the total load comprising linear forces and tangential torques.

Key words: object shape; grasp stability; fingertip forces; human hand; precision grip; fingertip torque; grip force; friction; rotational slip; tangential torsional loads
We use our digits to manipulate objects with widely diverse properties in a variety of dexterous maneuvers and readily maintain stable grasps by controlling against accidental slips. There have been many studies of the control of grasp stability during manipulation in which the object had flat grasp surfaces, and the destabilizing loads primarily involved linear load forces tangential to the grasp surfaces. Approximately 15 years ago, it was demonstrated that when objects are lifted using a precision grip between the tips of the index finger and thumb, subjects maintain grasp stability by automatically changing the normal grip forces applied to the grasp surfaces in parallel with changes in the applied tangential load forces (Johansson and Westling, 1984; Westling and Johansson, 1984). Thus, the grip force in each instance is constrained by the active sensorimotor program to vary in proportion to the load force. This linkage of forces, which is expressed in a variety of grasp configurations and tasks involving tangential loads of various complexities, ensures that appropriate grip forces are applied to prevent frictional slips, irrespective of the tangential load (Johansson and Westling, 1988a,b; Flanagan and Wing, 1993, 1995, 1997; Flanagan and Tresilian, 1994; Kinoshita et al., 1996; Burstedt et al., 1997). Moreover, based on tactile information, humans adjust the ratio between the grip and load forces to the prevailing friction to maintain an

\footnotetext{
Received Aug. 3, 1998; revised Sept. 30, 1998; accepted Oct. 2, 1998.

This study was supported by Swedish Medical Research Council Project 08667, Department of Naval Research Grant N00014-92-J-1919, and the Göran Gustafsson Foundation for Research in Natural Sciences and Medicine. Antony W. Goodwin was supported by the University of Melbourne. We thank Dr. G. Westling and L. Näslund for technical support.

Correspondence should be addressed to A. W. Goodwin, Department of Anatomy and Cell Biology, University of Melbourne, Parkville, Victoria 3052, Australia. Copyright (C) 1998 Society for Neuroscience 0270-6474/98/1810724-11\$05.00/0
}

adequate safety margin against frictional slips (Johansson and Westling, 1984, 1987; Cadoret and Smith, 1996). In common tasks, the loads that potentially destabilize grasp typically include torques tangential to the grasp surfaces. For example, in a precision grip task, tangential torques occur whenever we tilt an object around a grip axis (line joining the fingertips) that does not pass through the center of gravity of the object. Tangential torques can also arise because the normal force is distributed across the skin-object contact area rather than being focused at a point (Buss et al., 1996; Howe and Cutkosky, 1996). In a "rotational slip and hold" task in which a single digit generated tangential torsional friction that stabilized a disk subject to torque loading, Kinoshita et al. (1997) showed that such a load dramatically affected the grip forces required to prevent slip. Furthermore, to perform this particular task, primarily designed to measure torsional friction, subjects regulated the grip forces to the tangential torque. However, it is not known whether subjects regulate the grip forces to torques tangential to the grasp surfaces that develop when hand-held objects are maneuvered in common natural tasks.

The above findings are all based on studies in which the manipulated objects had flat grasp surfaces, but most objects that we handle in everyday activities have grasp surfaces that are curved. We have demonstrated recently that when the grasp is subjected to linear load forces, surface curvature substantially influences neither used fingertip forces nor the minimum grip force required for grasp stability (Jenmalm et al., 1998). It is not known whether this also applies to conditions in which the fingertip loads involve torques tangential to the grasp surfaces. It may be that changes in curvature influence the rotational friction more than the linear friction and that torsional and linear finger- 

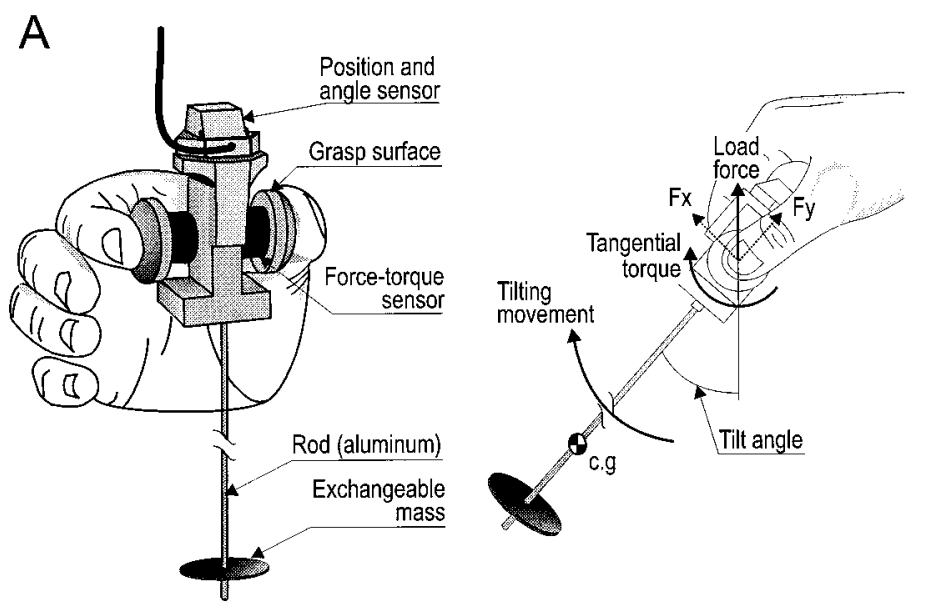

B

\section{Surface curvatures $\left(\mathrm{m}^{-1}\right)$}
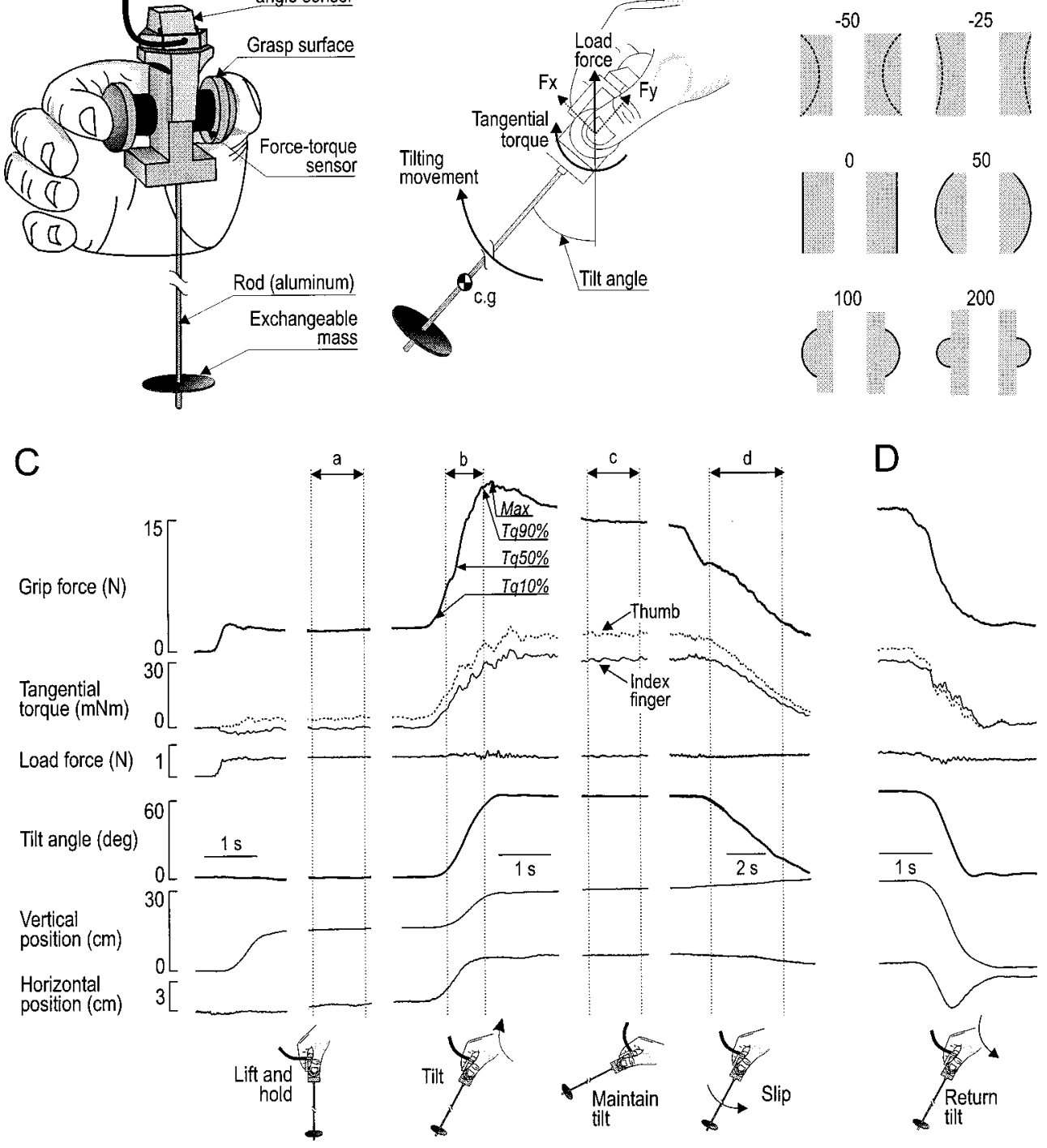

Figure 1. Description of the task. A, Front view of the object held in the vertical position and side view of the tilted object. The load force is the vector sum of the forces $\mathrm{F}_{\mathrm{x}}$ and $\mathrm{F}_{\mathrm{y}} ; c . g$, center of gravity. $B$, Side view of the six spherically curved grasp surfaces. $C$, Time traces of the major parameters measured during the various phases of a trial in the first experiment. Grip force is averaged for the two digits; tangential torque is shown independently for the thumb (broken line) and index finger (solid line); load force is averaged. The measurements representing the static hold phase before tilting are shown by $a$ (1 sec period), the tilting phase is shown by $b$, the static tilt phase is shown by $c$ ( 1 sec period), and the slip test is shown by $d$. Curvature, $50 \mathrm{~m}^{-1}$; target torque, 66 mNm. $D$, In the second experiment, there was no slip test; instead, the object was returned to its holder. Curvature, $100 \mathrm{~m}^{-1}$; target torque, $74 \mathrm{mNm}$.

tip loads may require different regimens in terms of grip force control. Although the viscoelastic fingertip will, to some extent, mold to the curvature of the surface of an object, changes in surface curvature are likely to influence the effective contact area that is relevant for torsional friction. With a marked convex surface, the torque-related tangential frictional forces are likely to be distributed over a smaller contact area than with a flat or concave surface, and thus the required grip forces may be substantially increased.

In this study, we first analyze how subjects control the grip forces to match changes in tangential torque as they develop in a highly natural task, i.e., when subjects grasp an object between the thumb and index finger, lift it, and then rotate it about the grip axis. We then explore possible effects of the curvature of the surface of an object on the coordination between grip forces and torque and the extent to which these effects reflect changes in the coefficient of rotational friction with changes in surface curvature. To this end, we examined a range of curvatures, extending from spherically concave grasp surfaces (radii, $20 \mathrm{~mm}$ ) for large digitobject contact areas to markedly convex surfaces (radii, $5 \mathrm{~mm}$ ) for small contact areas.

\section{MATERIALS AND METHODS}

\section{Subjects}

Eight right-handed healthy human volunteers (three females and five males) ranging in age from 24 to 53 years participated in these experiments. Informed consent was obtained from all subjects, and the local ethics committee approved the experimental protocol. Approximately 5 min before the experiments commenced, the subjects washed their hands with soap and water. During the experiments, they sat in an office chair with the right upper arm parallel to the trunk and the forearm extended. A test object, resting in a holder on the floor, had its grasp surfaces located $35 \mathrm{~cm}$ above the ground and $\sim 10 \mathrm{~cm}$ to the right of and $20 \mathrm{~cm}$ anterior to the subject's right hip. Subjects grasped the object in a precision grip using the right index finger and thumb, lifted the object, and then tilted it through $\sim 65^{\circ}$ by principally using a combination of elbow flexion and radial flexion of the wrist. The subjects could see the object and their hand throughout the experiments.

\section{Experimental setup}

The test object had two symmetrical grasp surfaces and a $31 \mathrm{~cm}$ long aluminum rod that protruded orthogonal to the axis between the centers of the grasp surfaces (Fig. 1A). Pairs of exchangeable matching grasp surfaces were attached to the test object. All surfaces were spherically curved (Fig. 1B); two pairs were concave with radii of 20 and $40 \mathrm{~mm}$ (curvatures, -50 and $-25 \mathrm{~m}^{-1}$ ), one was flat (curvature, $0 \mathrm{~m}^{-1}$ ), and 


\begin{tabular}{|c|c|c|c|c|c|c|c|c|}
\hline \multirow{2}{*}{$\begin{array}{l}\text { Surface } \\
\text { curvature } \\
\left(\mathrm{m}^{-1}\right)\end{array}$} & \multicolumn{8}{|c|}{ Target torque, nominal values (mNm) } \\
\hline & 31 & 44 & 55 & 66 & 88 & 114 & 156 & 189 \\
\hline-50 & & & & $65 \pm 2.7$ & & $112 \pm 3.0$ & $154 \pm 3.5$ & $187 \pm 5.0$ \\
\hline-25 & & & & $65 \pm 2.9$ & & $113 \pm 3.3$ & $154 \pm 3.8$ & $187 \pm 3.5$ \\
\hline 0 & & & & $65 \pm 2.7$ & & $112 \pm 3.1$ & $155 \pm 4.1$ & $188 \pm 5.4$ \\
\hline 50 & & & & $65 \pm 2.4$ & $88 \pm 3.0$ & $114 \pm 3.1$ & $155 \pm 5.1$ & \\
\hline 100 & & $43 \pm 2.7$ & & $65 \pm 2.8$ & $87 \pm 3.0$ & $113 \pm 4.0$ & & \\
\hline 200 & $31 \pm 2.8$ & $43 \pm 2.7$ & $54 \pm 3.4$ & $65 \pm 2.8$ & & & & \\
\hline
\end{tabular}

For each of the six surfaces, four different exchangeable masses were used, resulting in four target torques in experiment 1. Torques $(\mathrm{mNm})$ shown in the table are the mean \pm SD over all trials and all subjects. The mean values differ slightly for different curvatures and differ slightly from the nominal values given in Materials and Methods because of the location of the contact points (see Materials and Methods) and variation in the tilt angle about the nominal value of $65^{\circ}$. In experiment 2 , the nominal target torque was $74 \mathrm{mNm}$, and the actual torque used was $73 \pm 2.6 \mathrm{mNm}$. The (nominal) target torque of $66 \mathrm{mNm}$ was used with all curvatures. The bold entries indicate a block of 4 curvatures $\times 3$ torques used in ANOVA.

three were convex with radii of 20,10, and $5 \mathrm{~mm}$ (curvatures, 50, 100, and $\left.200 \mathrm{~m}^{-1}\right)$. Surfaces were coated with silicon carbide grains $(50-100 \mu \mathrm{m})$ covered with a thin layer of cyanoacrylate to give a finish similar to that of a fine grain sandpaper. When attached, the lateral edges of the surfaces were separated by $59 \mathrm{~mm}$.

Each surface was attached to the object via a six-axis force-torque sensor (Nano F/T transducer; ATI Industrial Automation, Garner, NC) that measured forces and torques in three dimensions. Grip force was measured along the grip axis defined by the line through the centers of the two grasp surfaces, and two force components were measured orthogonal to the grip axis, oriented along the long axis of the object $\left(\mathrm{F}_{\mathrm{y}}\right)$ or at right angles to it $\left(\mathrm{F}_{\mathrm{x}}\right)$. The vector sum of these components was defined as the load force, the major component of which supported the weight of the object. Grip forces were measured with a resolution of 0.05 $\mathrm{N}$, and $\mathrm{F}_{\mathrm{y}}$ and $\mathrm{F}_{\mathrm{x}}$ were measured with a resolution of $0.025 \mathrm{~N}$. Torques were measured about the three orthogonal force axes, intersecting at the center of the grasp surface, with a resolution of 0.125 milli-Newtonmeters $(\mathrm{mNm})$; torques about the grip axis corresponded to the tangential torques applied to the grasp surfaces by the contacting digits. An electromagnetic position sensor (FASTRAK; Polhemus, Colchester, VT) was attached to the object to measure its vertical position, its horizontal position in the anteroposterior direction, and its angle of tilt in relation to the vertical. In the environment of operation of the sensor, the linearity of the position measurement was better than $0.5 \mathrm{~mm}$ (resolution, $0.12 \mathrm{~mm}$ ) and the linearity of the angle measurement better than $1^{\circ}$ (resolution, $0.025^{\circ}$ ).

The rod extending from the object allowed exchangeable masses to be placed $32 \mathrm{~cm}$ below the grip axis. The resulting center of gravity of the object varied with the magnitude of the added mass and was between 4 and $14 \mathrm{~cm}$ below the grip axis when the rod was oriented vertically. Rotating the object about the grip axis thus produced torques tangential to the grasp surfaces. Nine different masses were used to arrive at the following target torques when the tilt angle was $65^{\circ}: 31,44,55,66,74,88$, 114,156 , and $189 \mathrm{mNm}$. The corresponding masses of the object were 88 , $93,96,104,108,111,121,136$, and $147 \mathrm{gm}$, respectively, and the load forces applied to support the object were thus $0.86,0.91,0.94,1.02,1.06$, $1.10,1.19,1.33$, and $1.44 \mathrm{~N}$, respectively. The aim of the long aluminum rod was to produce large target torques with small linear load forces and to minimize the errors resulting from variation in the contact points between the digits and the curved surfaces as detailed below. An increase in target torque was accompanied by only a small change in the vertical load force on the digits (target torque increased by a factor of 6.1, whereas the mass of the object increased by a factor of 1.7). From the data of Kinoshita et al. (1997), the linear force loads observed in our experiments (generally $<1 \mathrm{~N}$ ) would have had negligible influences on grip force requirements compared with those imposed by the torques used.

\section{Experimental procedure}

Each subject performed two experiments. In the first experiment, a trial consisted of the following temporal sequence of events. (1) The subject lifted the object vertically from its support through a few centimeters and held it in a stationary position with the rod oriented vertically. (2) After $5 \mathrm{sec}$, a $1 \mathrm{kHz}$ tone signaled the subject to tilt the object through $65^{\circ}$ by rotating it around the grip axis; the tone ceased when the target angle was reached. For additional guidance, the target angle was indicated by a line drawn on a board located in a sagittal plane $\sim 10 \mathrm{~cm}$ to the right of the subject's right arm. (3) The object was held in the tilted position for $4 \mathrm{sec}$; this phase was termed the static tilt phase. (4) Then, an auditory cue $(1 \mathrm{kHz}$ tone for $0.1 \mathrm{sec})$ signaled commencement of a rotary "slip test" in which the subject decreased the grip force slowly and allowed the object to rotate smoothly back to a vertical orientation, under the influence of gravity. (5) After this slip test, the subject replaced the object in its support. During phase 3, the subject was required to maintain the tilt angle between 60 and $70^{\circ}$ for at least $4 \mathrm{sec}$. If these bounds were exceeded, which happened occasionally, an auditory cue indicated that a correction was required, and the 4 sec static tilt interval was reset. Each trial lasted for $\sim 20 \mathrm{sec}$, and a break of $\sim 10 \mathrm{sec}$ was left between trials. A test series comprised 24 trials and consisted of six blocks of four trials each. In each block, one of the six surface curvatures was used, and in the four successive trials, four selected exchangeable masses were used, resulting in four different nominal target torques. The six surfaces and the four torques for each surface were presented in a random order. Three such series were run on each subject. Table 1 shows the four target torques used for each of the six curvatures. As seen in Table 1, the tangential torques during phase 3 varied slightly from trial to trial because of variation in the tilt angle and in the location of the contact points; in the remainder of the paper, target torques are given as the nominal values (that would have resulted from the nominal tilt of $65^{\circ}$ ). The selection of target torques for each pair of surface curvatures was such that the range of grip forces used was comparable for all surfaces, as determined in pilot experiments.

The second experiment comprised a single series of 39 trials consisting of 13 blocks of three trials each. A single mass was used corresponding to a target torque of $74 \mathrm{mNm}$. In the odd blocks, the grasped surfaces were flat (curvature, $0 \mathrm{~m}^{-1}$ ) for all three trials, and in the even blocks, the curvature was $100 \mathrm{~m}^{-1}$. In this series, the subject initially lifted and tilted the object in an identical manner to phases 1-3 in the first experiment. Then, in phase 4 , after maintaining the tilt for $4 \mathrm{sec}$, an auditory cue signaled the subject to rotate the object back to the vertical orientation (instead of performing the rotational slip test) by a combined elbow extension and ulnar flexion of wrist. In phase 5, the object was held in this position for another $5 \mathrm{sec}$ after which, after an auditory cue, the object was replaced in its support.

Before the experiments, each subject was specifically instructed to grasp the surfaces at their centers, and the experimenter pointed out the center of one convex surface and one concave surface. After the experimenter had demonstrated the task, the subject performed five practice trials using the flat surfaces with a mass resulting in a target torque of 66 $\mathrm{mNm}$. The subjects performed the tilting movement at their preferred speed. During all experiments, the subject was observed carefully to ensure compliance with instructions.

\section{Data collection and analysis}

All data were digitized using a flexible data acquisition and analysis system (SC/ZOOM; Department of Physiology, Umeå University, Umeå, Sweden) and stored on a personal computer. Signals were recorded with a resolution of 12-bits (400 samples/sec) from the force- 
torque sensors and 14-bits (120 samples/sec) from the position-angle sensor.

If the only tilt of the object were that produced by rotation about the grip axis (as was requested of the subjects), then the grip force would have been the same for the thumb as for the index finger. Observation of our subjects revealed no obvious violation of this instruction; over all trials and all subjects, the ratio of the two grip forces during phase 3 above was $1.01 \pm 0.01$ (mean $\pm \mathrm{SD} ; n=888$ ). Thus, in Results, we present data for the average grip force of the two digits. Tangential torques are either specified for each digit independently or are given as the total torque (sum for the two digits) as appropriate.

Time traces of the measured parameters are illustrated for the various phases of the task in Figure $1 C$. For each trial, the grip forces, tangential torques, linear load forces, and tilt angle were measured as their mean values during a $1 \mathrm{sec}$ interval when the object was held steady before the tilting movement (Fig. $1 C, a$ ). This interval commenced $4 \mathrm{sec}$ after the object was first contacted (defined by the time at which the sum of the grip forces for the two digits first exceeded $0.3 \mathrm{~N}$ ). Mean values of the same signals were also calculated during a $1 \mathrm{sec}$ interval, located in the middle of the $4 \mathrm{sec}$ static tilt phase (Fig. $1 C, c$ ).

During the tilt phase (Fig. $1 C, b$ ), grip force measurements were taken at times when the total tangential torque at the two digits had increased by 10,50 , and $90 \%$ of the increase in total tangential torque that occurred from the period before tilt (Fig. $1 C, a$ ) to the period when the object was maintained tilted (Fig. $1 C, c$ ). In addition, the maximum grip force was measured as the peak value within $\pm 1 \mathrm{sec}$ after the end of the tilt phase. Angular velocity and angular acceleration were assessed from the first and second time derivatives of the tilt angle, respectively, using a \pm 6 point numerical differentiation $( \pm 50 \mathrm{msec}$ window). The rise time of the angle during the tilting movement (Fig. $1 C, b$ ) was taken as the interval from 10 to $90 \%$ of the increase in tilt angle that occurred from phase $a$ to phase $c$ in Figure $1 C$.

For each trial in the first experiment, the relationship between grip force and tangential torque over the window of rotational slip (Fig. $1 C, d$ ) was established independently for each digit by linear regression. This window was determined off-line by visual inspection of the time traces of tilt angle, grip force, and tangential torque and by inspection of the corresponding force-torque plots. The regression constants and the measured tangential torques were used to estimate, for each digit, the minimum grip forces required to prevent slip (slip forces) during the static tilt phase. The safety margin was calculated as the difference between the static grip force and the larger of the slip forces for the thumb and index finger.

Grasp points and errors in torque measurements. Subjects were instructed to grasp the surfaces of the test object at their centers. Although they performed remarkably well in this respect, there were small deviations that introduced errors in the target torque, as well as in our estimates of the true torques, applied by the digits. First, subjects tended to displace the grip axis toward the center of mass of the object, decreasing the effective length of the lever arm by $\sim 2 \mathrm{~mm}$ and thereby decreasing the target torque (at a tilt angle of $65^{\circ}$ ) in relation to its nominal value (cf. Table 1). Second, if the grasp points deviated from the axis intersecting the centers of the grasp surfaces, the torque measured about this axis could have differed from the true torques at the fingertips. That is, linear load forces (orthogonal to this axis) could have contributed to the measured torque. These linear forces resulted from the vertical forces applied to counterbalance the weight of the object and from "internal" forces applied to prevent the object from spinning if the grasp points were located asymmetrically on the grasp surfaces. The linear forces were measured and were only of the order of $1 \mathrm{~N}$. Moreover, when the object was held tilted, the deviation of the equivalent point of contact between the digit and the surface, from the center of the surface in the direction along the lever arm of the object, was $3.2 \pm 2.3 \mathrm{~mm}$ for the thumb and $2.0 \pm 2.1 \mathrm{~mm}$ for the index finger (mean $\pm \mathrm{SD}$; data pooled across all trials; location of contact points calculated as in $\mathrm{Ki}$ noshita et al., 1997). In the direction orthogonal to the lever arm, the deviation was $0.0 \pm 1.9 \mathrm{~mm}$ for the thumb and $1.7 \pm 2.1 \mathrm{~mm}$ for the index finger. From these deviations and the magnitude of linear forces, we can safely conclude that the errors in torque measurements were small in relation to the magnitudes of the target torques. In fact, they were of the same order of magnitude as variations in torque resulting from variations in the effective length of the lever arm.

Statistical analysis. Pearson product-moment correlations and repeated-measures ANOVA were used to evaluate the influence of tangential torque, surface curvature, and digit; details are provided in Results. The level of probability selected as statistically significant was $p<0.05$. All reported correlation coefficients were significant. Unless otherwise stated, where data were pooled across subjects the following procedure was used. For each subject, the trials in which the experimental parameters (target torque and surface curvature) were identical were combined, providing a subject mean for each measurement. Values for these "average trials" were used in the ANOVA analyses, and in many figures subject means and SEM $(n=8)$ are presented.

\section{RESULTS}

The results are presented in three sections. In the first, we characterize the task based on data for which the grasp surfaces were flat and the target torque was held constant. In the second, we explore the effects of changing target torque for flat surfaces. Finally, we analyze the effects of changing both the curvature of the grasped surfaces and the target torque. We stress that the grasp behavior observed and described here emerged automatically in the sense that subjects were not given any instructions pertaining to grip force.

\section{Force-torque coordination with flat parallel grasp surfaces}

When the subject tilted the test object by flexing the elbow and wrist, the resulting increase in tangential torque at the digits was accompanied by an increase in the grip force with no apparent time lag. In Figure $2 A$, the subject tilted the object at a moderate speed. There was a continuous increase in the torque at each digit accompanied by a coordinated parallel increase in the grip force. Figure $2 E$, which shows the grip force plotted against the tangential torque at each digit, emphasizes their coordination throughout the tilting movement. In Figure 2, $B$ and $F$, the subject tilted the object rapidly (see angular velocity in $B$ ) and the torques generating the tilt showed a more complex profile. During the initial phase of the tilt, the grip force increased in parallel with the applied torques until they reached their initial peaks associated with maximum angular acceleration of the object (Fig. 2B, $F$, arrowheads). After the peak angular velocity of the object, its angular deceleration was caused by a marked decrease in the torque drive, with minimum values close to zero. Despite this decrease in torque, the grip force continued to increase, and when the "ringing" in torque had ceased, the grip force had reached a value close to that maintained during the subsequent static tilt phase. That is, the grip force was coordinated to the overall increase in torque but did not change with the rapid torque fluctuations during the tilting movement. Accordingly, the ratio of grip force to tangential torque decreased markedly during the initial torque peak and then increased during the subsequent dip in torque. Interestingly, if rotational slips occurred at the minimum force-to-torque ratio (Fig. $2 F$, arrowhead), they would have occurred when the object had a high positive angular velocity so that during such a slip, the tilting movement would have continued.

When the object was returned at a moderate speed to its original vertical orientation (second experiment), the grip force and torques decreased smoothly and in parallel throughout the replacement phase (Fig. $2 C, G$ ). In trials with a rapid rotation of the object back to the vertical position (Fig. 2D, H), the angular movement was initiated by a sharp decrease in tangential torques at the digits, lasting until the angular deceleration of the object was maximal. This decrease was coupled with a parallel decrease in grip force. Then, the subject increased the torques to brake the angular deceleration, whereas the grip force continued to decrease. After the trough in torque terminated by a local peak (Fig. $2 D, H$, arrowheads), the torques and grip force decreased together 


\begin{abstract}
Figure 2. Coordination of force and torque illustrated for selected single trials in experiment 2. $A-D$, Time traces showing (from top to bottom) the average grip force, the total torque on the digits (solid line), the torque predicted from regression (thin line), the torque on each digit, the angle of tilt, and the first and second time derivative of the tilt angle. $E-H$, Plots of grip force versus torque for each digit. In $A$ and $E$, the object was tilted slowly (rise time, $0.90 \mathrm{sec}$ ). In $B$ and $F$, the tilt was more rapid (rise time, $0.41 \mathrm{sec}$ ). In $C$ and $G$, the object was returned to the vertical slowly (fall time, $1.11 \mathrm{sec}$ ). In $D$ and $H$, the return was more rapid (fall time, $0.38 \mathrm{sec}$ ). The regression predicting torque had correlation coefficients of $0.997,0.996,0.998$, and 0.996 , in $A, B, C$, and $D$, respectively. Target torque was $74 \mathrm{mNm}$, and grasp surfaces were flat.
\end{abstract}
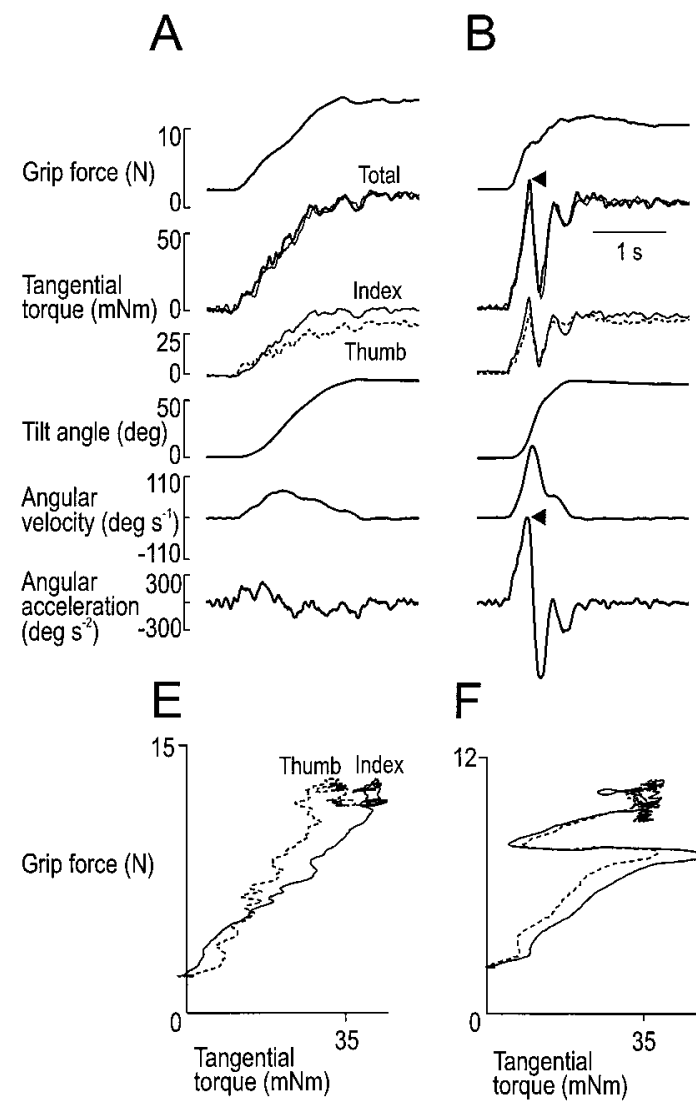
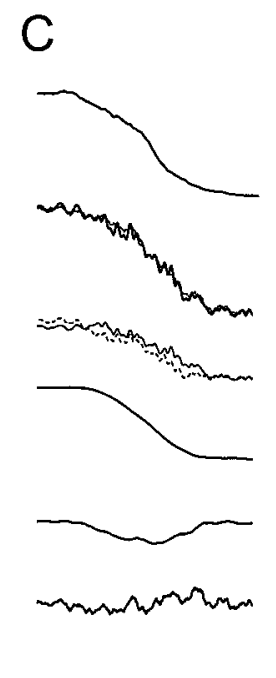

G

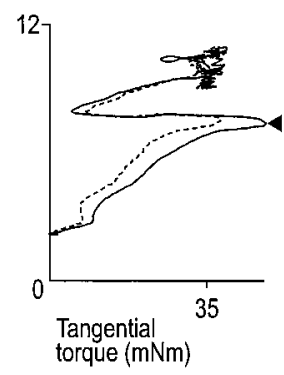

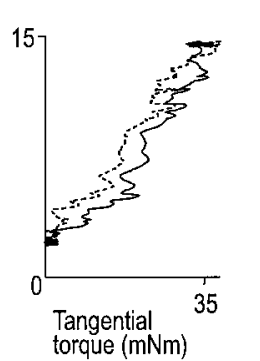

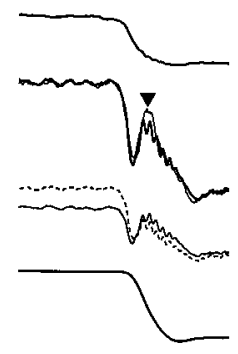

D

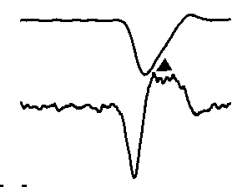

$\mathrm{H}$

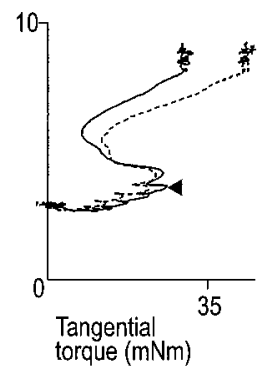

until the object was oriented vertically. Thus, during the replacement of the object, there could also be a temporary decoupling of grip force and torques, but the grip force was coordinated with the overall change in torque. Interestingly, as judged from the ratio of grip force to tangential torque, potential rotational slips would have occurred close to the minimum angular velocity and not in the final phase of the replacement (Fig. 2D, H, arrowheads).

For object rotation at any speed, the trajectory of the tilt angle was close to critically damped. A simple mechanical model that predicted the time-varying torque from the motion of the object verified that the trajectory of the tilt angle resulted from the torque dynamics. We used the following linear regression model: $T=a+b \sin \alpha+c \alpha^{\prime \prime}$, where $T$ is the total torque (sum of the torque at each digit); $a, b$, and $c$ are regression coefficients; $\alpha$ is the tilt angle; and $\alpha^{\prime \prime}$ is the angular acceleration. In mechanical terms, the expressions $\sin \alpha$ and $\alpha^{\prime \prime}$ account for the static torque and the moment of inertia of the object, respectively. Torques resulting from linear acceleration of off-axis mass in the vertical and anteroposterior directions were negligible because of the relatively small accelerations. Likewise, torques resulting from off-axis load forces (grasp points not at the centers of the surfaces) were negligible and did not improve the regression significantly. In Figure 2, $A-D$, the time traces representing the total torque on the digits show the recorded torques (solid lines) and the torques predicted from the regression (thin lines) for representative examples of single trials. Because the model is a direct consequence of Newton's Laws of Motion, the fit was very good with coefficients of correlation close to unity (i.e., $>0.99$ for all trials).

The speed of the tilting movement varied between the first and the second experiments; in the first experiment, each trial was terminated by the slip test, whereas in the second experiment, the subject rotated the object back to the vertical orientation in an ordinary manner. The rise times of the tilt angle in the first experiment were $1.05 \pm 0.41$ (mean $\pm \mathrm{SD} ; n=96$ ) and in the second $0.61 \pm 0.22$ ( $n=168 ; p \ll 0.001$; Mann-Whitney $U$ test $)$. The example in Figure 2, $A$ and $E$, illustrates a trial in which the rise time of the tilt was representative of the trials used by our subjects in the first experiment, and Figure 2, $B$ and $F$, illustrates one of the fastest trials that was observed in the second experiment. The fall times in the second experiment were $0.76 \pm 0.37$ $(n=312)$.

\section{Effect of target torque}

In the first experiment, four different exchangeable masses were used, with the flat grasp surfaces resulting in four different target torques. The effect of different torques on the coordination between grip force and tangential torque is illustrated for single trials in a single subject in Figure 3, $A$ and $B$. An increase in target torque resulted in an increase in grip force and, for all four torques, the instantaneous grip force was coordinated to the instantaneous torque throughout the tilting movement. The trajectories for the higher torques in Figure $3 B$ overlie the trajectories for the lower torques, indicating a consistent force-torque relationship, regardless of final torque. This pattern was consistent for all subjects and is illustrated for an additional three subjects in Figure 3, $C-E$. A second feature seen in Figure $3 A$ is that the rise time of the tilting movement did not vary with changes in the target torque (see the consistency of the angle traces). This was true for each subject, but the mean rise time varied between individuals (range of mean values, 0.7-1.8 sec). 

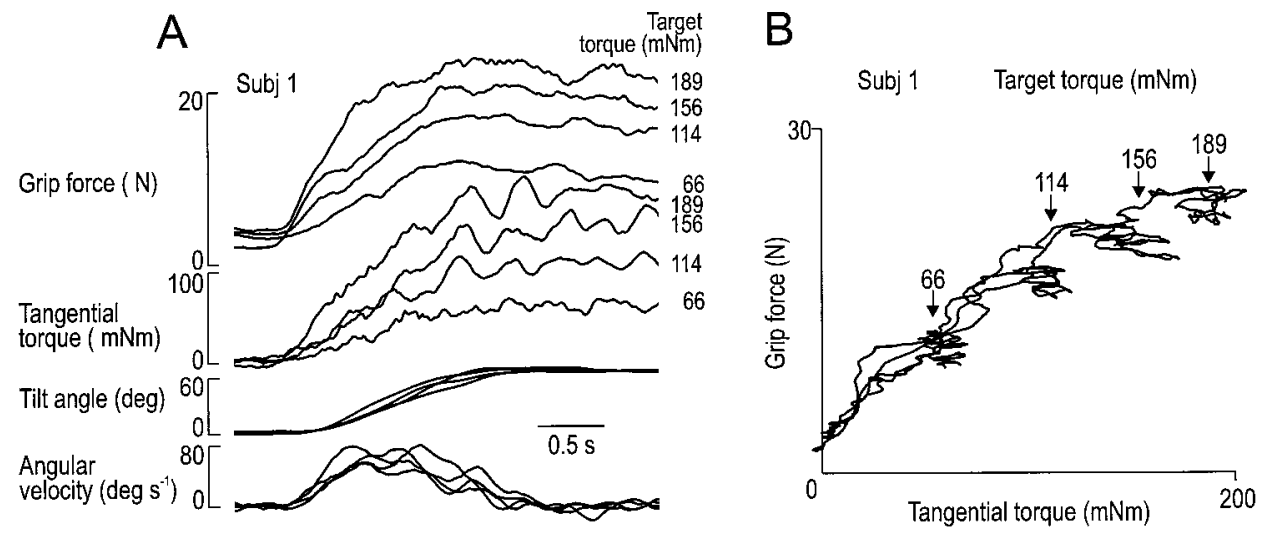

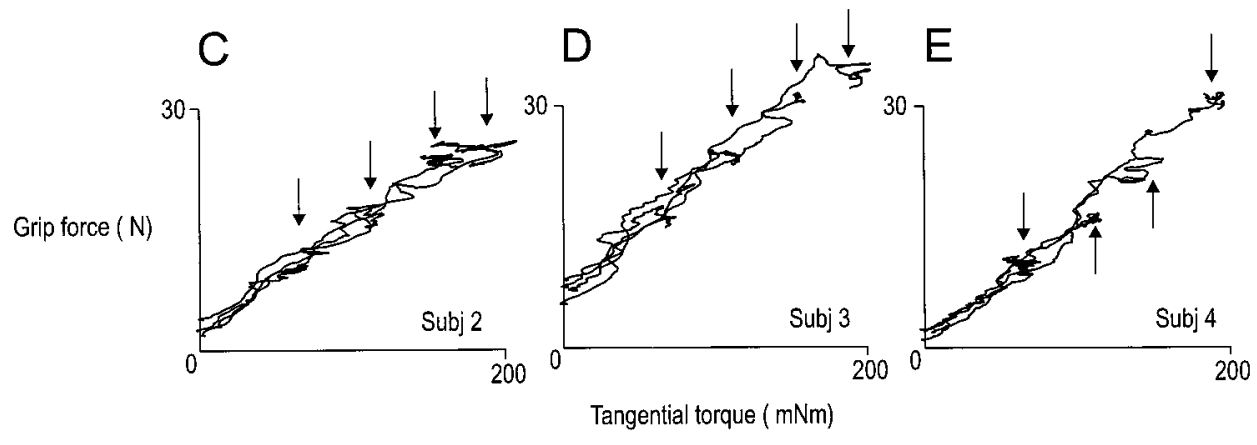

A

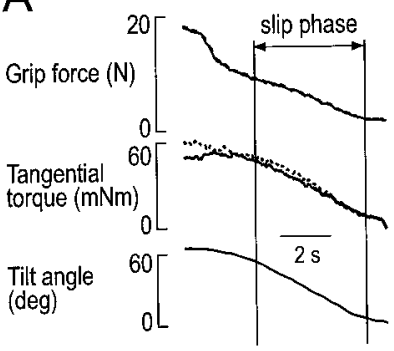

$\mathrm{B}_{\text {(index) }}$

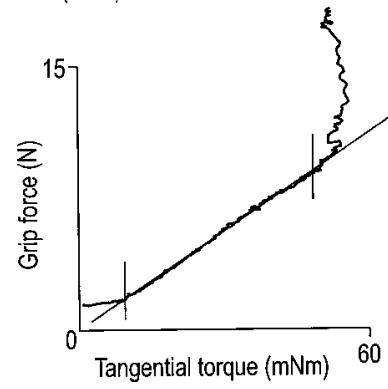

$C_{\text {(thumb) }}$

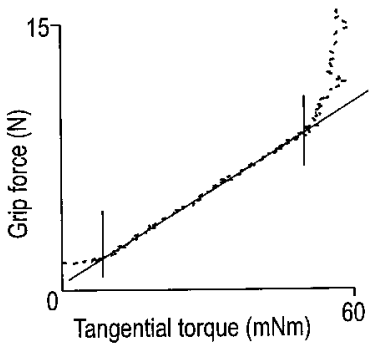

Figure 3. Effect of target torque on forcetorque coordination with flat grasp surfaces. $A$, Time traces for average grip force and total tangential torque on the digits, the angle of tilt, and the first time derivative of the tilt angle during four trials for subject 1 . The four trials were aligned at the time when tilt angle was $10^{\circ} . B$, Force-torque plots of the same data as in $A$. $C-E$, For three other subjects, the force-torque coordination is shown for four single trials with the four target torques $(66,114,156$, and $189 \mathrm{mNm}$ ) indicated by the arrows.

Figure 4. Rotational slip illustrated for a single trial in a single subject with flat surfaces and a target torque of $66 \mathrm{mNm}$. Thin vertical lines demarcate the slip phase. $A$, Time trace of average grip force, tangential torque on the thumb (broken line) and index finger (solid line), and tilt angle. B, Forcetorque plot (thick line) and linear regressions (thin line) during slip for the index finger. Correlation coefficient is 0.999 , slope is 0.185 , and intercept is 0.134 . C, Forcetorque plot (broken line) and linear regressions (thin line) during slip for the thumb. Correlation coefficient is 0.999 , slope is 0.171 , and intercept is 0.333 .
Accordingly, there was no statistically significant effect of target torque on rise time $(p=0.246)$.

\section{Safety margin preventing rotational slips}

When the object was tilted toward the target orientation of $65^{\circ}$ and held tilted in this position, there were no obvious accidental rotational slips between the object and the fingertips. Apparently, the grip force used was greater, by some safety margin, than the minimum force required to prevent slip, termed the slip force. The slip force is determined by the tangential torque, the coefficient of rotational friction, the linear load force, the coefficient of linear friction, and to some extent by interactions between the effects of torque and load force (Kinoshita et al., 1997). In the present experiments, the linear load forces were relatively small and would have contributed little to the slip force when the object was held tilted (cf. Kinoshita et al., 1997).

\section{Rotational slip coefficient}

A rotational slip coefficient for each digit was determined as follows. At the end of the static tilt phase, the subject reduced the grip force slowly, allowing the object to undergo slow rotational slip back to the vertical orientation. During the slip phase, indicated in Figure $4 A$, vertical lines, the force and torques decreased in parallel and, as seen from the angle trace, the object slipped smoothly back to the vertical orientation. For both digits, the relationship between grip force and tangential torque was linear during the slip phase (Fig. $4 B, C$ ). The slope of the regression line is termed the rotational slip coefficient and corresponds to the inverse of the coefficient of rotational friction used by Kinoshita et al. (1997). The fact that force and torque follow the same straight line throughout the slip phase shows that the rotational slip coefficient is independent of tangential torque. All subjects showed linear relationships comparable to those in Figure 4 for all four target torques with the flat grasp surfaces. The 192 (4 target torques $\times 3$ repetitions $\times 2$ digits $\times 8$ subjects) correlation coefficients ranged from 0.969 to 0.999. Although ANOVA showed that there was no significant difference between the coefficients for the two digits $(p=0.08)$ and no significant effect of target torque ( $p=0.16)$, we cannot exclude a modest difference in friction for the digits (Kinoshita et al., 1997).

The rotational slip coefficients were measured during a phase 


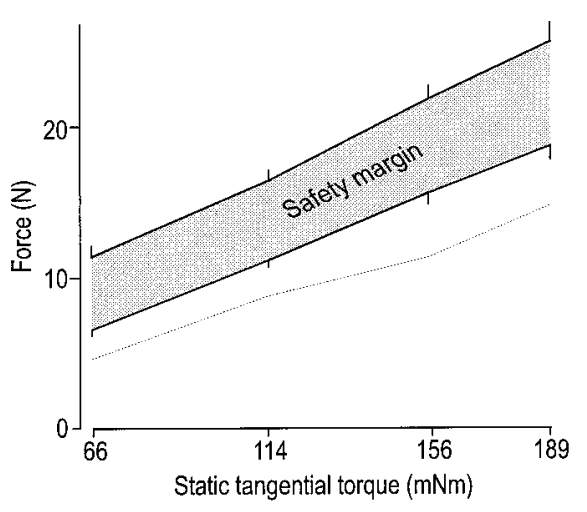

Figure 5. Safety margin in grip force during the static tilt phase for flat grasp surfaces. Data averaged over the eight subjects. Solid lines show grip force (mean + SEM) and critical force (slip force on the critical digit; mean - SEM). Broken line shows the slip force on the noncritical digit. Shaded area shows the safety margin (difference between grip force and critical force). Target torques were $66,114,156$, and $189 \mathrm{mNm}$.

of continuous slip in which the operating frictional coefficient would be a dynamic coefficient of rotational friction. Two factors indicate that in our experiments there was little difference between the static and dynamic coefficients of rotational friction. First, as grip force decreased to the slip point, the curve in the force-torque plot (Fig. 4B,C) at the onset of slip never dipped below the linear relationship, as would have occurred if the static rotational friction had exceeded the dynamic friction. Second, on some occasions, some of our subjects showed distinct slip-stick behavior during the slip test, but the relationship between the grip force and torque did not shift appreciably between the slip and stick phases.

\section{Safety margins}

The safety margin in grip force during the static tilt phase, when the object was held at an orientation of $65^{\circ}$, was estimated in each trial as follows. The slip force at each digit was estimated from the regression constants of the force-torque relationships obtained as described above and from the value of the static tangential torque. The digit that showed the greater of the two slip forces was termed the critical digit; rotational slip would have occurred if the grip force had been less than this slip force. The safety margin was defined as the difference between the grip force used by the subject and the slip force of the critical digit. In Figure 5, the grip and critical forces are shown for the four target torques. Safety margins, shown in Figure 5, shaded area, were approximately invariant with target torque $(p=0.066)$. It is also evident that, on average, the slip force on the noncritical digit was smaller than that of the critical digit.

In $53 \%$ of trials, the thumb was the critical digit. Partitioning of torque between the two digits was the major factor determining which digit was critical, because the rotational slip coefficients were similar, as indicated above. In the trials exemplified in Figure 2, the torques on the two digits were approximately the same, but the partitioning of torque varied widely. The ratio of torque on the thumb to torque on the index finger in the static tilt phase had a mean of 1.17 and a SD of 0.64 . In 80 of 96 cases, the digit with the higher static torque was the critical digit. In the remaining 16 cases, the ratio of torques on the two digits was close to 1 , in which case the slight difference between the rotational slip coefficient for the two digits determined the critical digit.

\section{Force-torque coordination with curved surfaces}

The grip force used by the subjects changed markedly with changes in the curvature of the grasp surfaces. Figure 6, $A$ and $B$, illustrates four single trials for one subject tilting the object with four different curvatures, all to the same target torque. For all curvatures, the grip force and the tangential torque increased in parallel throughout the tilt (Fig. 6B), but a change in surface curvature changed the balance between grip force and tangential torque throughout the trial. This parametric effect on the forcetorque coordination was already evident at the onset of the tilting movement. The force-torque coordination is shown for three other subjects in Figure 6, $C-E$. Changes in surface curvature had weak effects on the rise time of the tilt angle, which increased with increasing surface curvature. For a target torque of $66 \mathrm{mNm}$, the rise times at $-50 \mathrm{~m}^{-1}$ and $200 \mathrm{~m}^{-1}$ were $0.94 \pm 0.39$ and $1.20 \pm$ $0.46 \mathrm{sec}$, respectively (mean $\pm \mathrm{SD} ; n=24 ; p=0.001$ ).

The coordination of force and torque during the tilt phase was examined more closely by plotting the grip force at five successive time points during the tilt (Fig. $7 A$ ). The first three points were the times at which the total torque at the two digits had increased by 10,50 , and $90 \%$ of the increase in total torque that occurred during the tilting movement. The remaining two torques were the torque at peak grip force and the torque during the static tilt phase. For all six curvatures, the grip force increased in parallel with the tangential torque up to the peak torque, and the two variables had an approximately linear relationship. Throughout the tilt, an increase in curvature scaled the ratio of grip force to tangential torque upward; that is, the higher the surface curvature, the higher the grip force at any given tangential torque. As early in the trial as the point at which torque was $10 \%$ of the static value, there was a significant effect of surface curvature $(p<$ 0.001).

Grip forces during the static tilt phase pooled for all eight subjects are shown in Figure 7, $B$ and $C$, for all six surfaces; for each surface, four different levels of target torque were used (Table 1). Grip force was scaled by both target torque and surface curvature. Curvature significantly affected the grip force at the target torque of $66 \mathrm{mNm}$, which was common for all curvatures $(p<0.001)$. The effect on grip force was significant for both curvature and target torque in the block indicated by bold entries in Table 1 (curvature, $-50,-25,0$, and $50 \mathrm{~m}^{-1}$; torque, 66, 114, and $156 \mathrm{mNm} ; p<0.001$ in both instances). Because the curvature influenced the slope of the relationship between grip force and tangential torque, the interaction term of these two factors was also significant $(p<0.001)$.

\section{Effect of surface curvature on safety marg in}

Rotational slip coefficients for curved surfaces. For each pair of curved surfaces, the relationship between grip force and tangential torque during the slip phase of each trial was examined independently for the two digits. In all cases, the relationship was highly linear as in the illustration for the flat surfaces in Figure 4. The 1152 correlation coefficients ranged from 0.906 to 1.00 (mean $\pm \mathrm{SD} ; 0.993 \pm 0.009$ ). The rotational slip coefficient, given by the slope of the regression line, increased with increasing convexity of the surfaces (Fig. $8 A$ ). There was some variation between subjects in the magnitude of the coefficients, particularly for the highly curved convex surfaces. Rotational slip coefficients for all six curvatures and for all four target torques used at each curvature are shown for the index finger and thumb in Figure 8, $B$ and $C$, respectively (data averaged across subjects). Coefficients were smaller for the thumb than for the index finger, particularly 

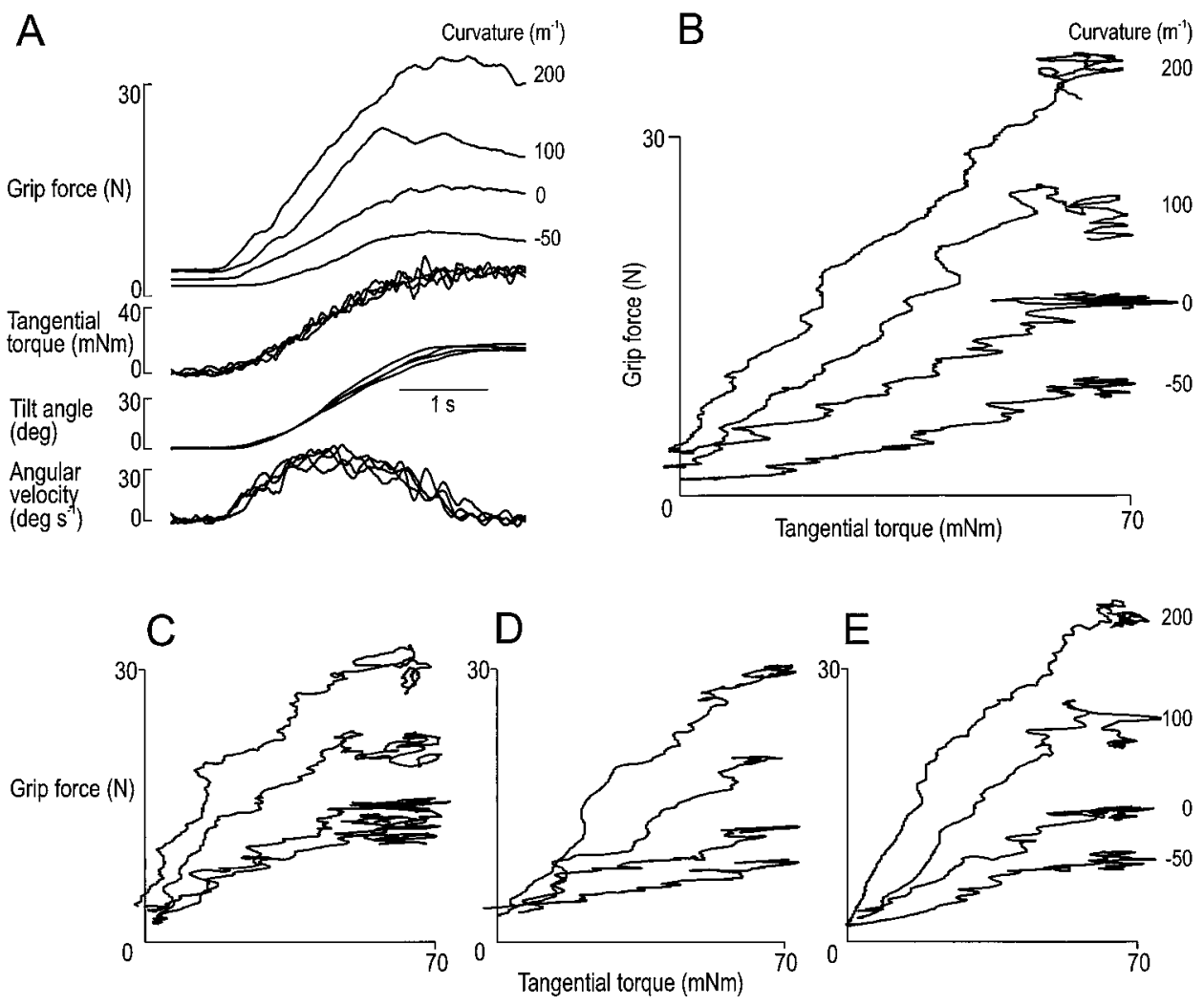

Figure 6. Coordination of mean grip force and total tangential torque with changes in surface curvature. $A$, Four single trials for one subject with a target torque of $66 \mathrm{mNm}$; superimposed trials were aligned at the time when tilt angle was $10^{\circ}$. The data illustrate the most concave surface (curvature, $\left.-50 \mathrm{~m}^{-1}\right)$, the flat surface $\left(0 \mathrm{~m}^{-1}\right)$, and the two most convex surfaces (100 and 200 $\left.\mathrm{m}^{-1}\right) . B$, Force-torque plots of the same data as in $A$. $C-E$, Comparable data for three additional subjects.

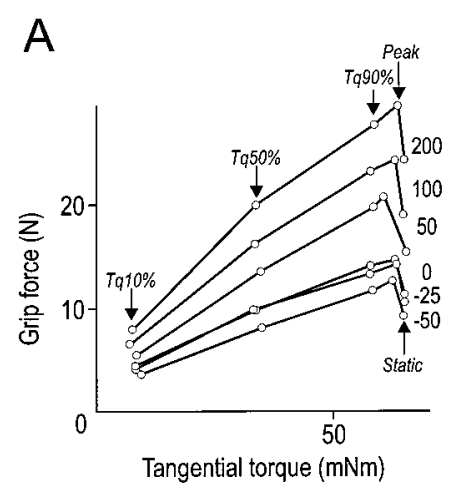

B

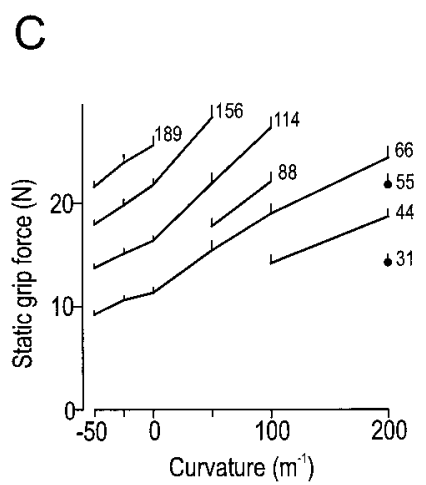

Figure 7. Effect of surface curvature and tangential torque on grip force. $A$, Coordination shown for time points during the tilt in which total tangential torque had increased to 10,50 , or $90 \%$ of the static tilt phase value, the torque at peak force, and the static torque. Average for all subjects $(n=8)$, with a target torque of $66 \mathrm{mNm}$. Parameters show surface curvature in units $\mathrm{m}^{-1} . B$, Mean + SEM grip force during the static tilt phase for the eight subjects. For each of the six surface curvatures (indicated by the parameters), there were four target torques (see Table 1 for details). $C$, For each of the eight target torques (values in milli-Newton-meters shown by the parameters), the number of curvatures tested varied from one (at 31 and $55 \mathrm{mNm}$ ) to six (at $66 \mathrm{mNm}$ ). Same data as in $B$ with the abscissa and parameter interchanged.

for the most concave and convex surfaces. Torque did not affect the slip coefficient (Fig. 8B,C). Two-way ANOVA showed a significant effect on rotational slip coefficient of curvature $(p<$ $0.001)$ and digit $(p=0.01)$.

Safety margin. This was defined as the difference between the grip force used by the subject during the static tilt phase and the corresponding slip force for the critical digit, as defined above. The safety margin is shown as a function of curvature in Figure $9 A$, hatched area, for a target torque of $66 \mathrm{mNm}$. Safety margin increased as the curvature of the surfaces increased $(p<0.001)$. In agreement with the data for flat grasp surfaces, at all curvatures the slip force on the noncritical digit was, on average, less than the slip force on the critical digit, primarily as a result of the unequal partitioning of the torque. Absolute grip force safety margins and relative safety margins (safety margins as fraction of grip force) are shown for all curvatures and torques in Figure 9, $B$ and $C$. In broad terms, absolute safety margin is more depen- dent on surface curvature than on target torque, whereas relative safety margin is more dependent on torque than on curvature. ANOVA indicated a reliable effect of curvature on absolute safety margin at a target torque of $66 \mathrm{mNm}(p<0.001)$ and of target torque on relative safety margin for the flat surface $(p<$ 0.001 ). For the block (curvature, $-50,-25,0$, and $50 \mathrm{~m}^{-1}$; torque, 66,114 , and $156 \mathrm{mNm}$; see Table 1, bold entries), the only significant effect was for target torque on relative safety margin.

Effect of previous surface curvature. A major reason for including experiment 2 in which the blocks (of three trials) alternated between surface curvatures of 0 and $100 \mathrm{~m}^{-1}$, all at a target torque of $74 \mathrm{mNm}$, was to examine whether coordination of grip force was influenced by the surface curvature used in the previous trial. For both surfaces, the test series included twelve trials for which the preceding tilt occurred with the same curvature (condition 1) and six trials for which the preceding tilt occurred with a different surface curvature (condition 2). To study the effect of 
Figure 8. Rotational slip coefficients for the six curved surfaces. $A$, For the target torque of $66 \mathrm{mNm}$, coefficients for the index finger are shown for each of the eight subjects. $B$, At each curvature, coefficients (mean over eight subjects) are shown for the index finger for each of the four target torques used. Note the overlap in data points for the four torques. Lines join points at common torques; for example, there are only two torques common to the surfaces at 100 and $200 \mathrm{~m}^{-1}$. $C$, As for $B$, except for the thumb. Details of the forces and torques are given in Table 1.

Figure 9. Safety margins used to prevent rotational slip. Data averaged over the eight subjects. A, Solid lines show grip force (mean + SEM; $n=8$ ) and critical force (slip force on the critical digit; mean SEM) for a target torque of $66 \mathrm{mNm}$. Shaded area shows safety margin (difference between grip force and critical force), and broken line shows slip force on the noncritical digit. $B$, Absolute safety margin $($ mean + SEM $; n=8)$ for all six curvatures (values in units $\mathrm{m}^{-1}$ indicated by the parameters). For each pair of surfaces, four target torques were used. $C$, Relative safety margins (absolute safety margin divided by grip force) for the six curvatures, each at four torques. Note the overlap in data points for the various surface curvatures. For $B$ and $C$, the combinations of curvatures and torques are given in Table 1.

the previous surface on force coordination, we compared five measures of grip force for the two conditions for both surfaces. The measures were the grip force at points at which the total torque at the two digits had increased by 10,50 , and $90 \%$ of the increase in total torque that occurred during the tilting movement, the peak grip force, and the static grip force (compare with Fig. 7A). There was no statistically significant effect of previous surface (i.e., no effect of "condition") on any of these measures for either of the two surfaces.

\section{DISCUSSION}

In the experiments reported here, we used a tilting task that is similar to natural maneuvers we use commonly. For such maneuvers, there may be large variations in tangential torques at the fingertips. We have shown that the sensorimotor system controlling grasp stability effectively adjusts the grip forces to match the demands imposed by varying torsional loads. Furthermore, we found that the required grip force was highly dependent on the curvature of the surface of the object and that this too was efficiently compensated for.

\section{Parallel coordination of grip force and tangential torque}

Throughout the tilt, tangential torque and grip force changed in parallel, with an approximately linear relationship. Thus, accidental rotational slips were prevented by the grip force increasing in parallel with the tangential torque. This coordination between grip force and torque resembles the coordination between grip force and linear load force that constitutes the basic strategy for the prevention of accidental linear slips (see introductory remarks). In particular, it resembles the coordination observed by Flanagan and Wing (1993) in which subjects transported a handheld object so that variations in load force arose from inertial

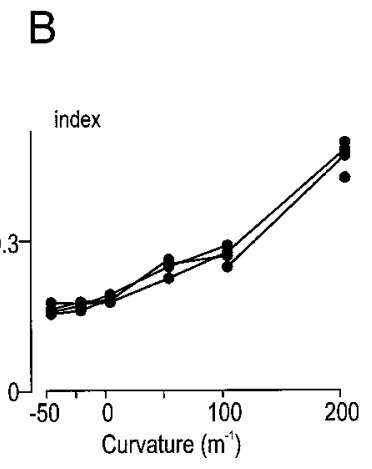

C

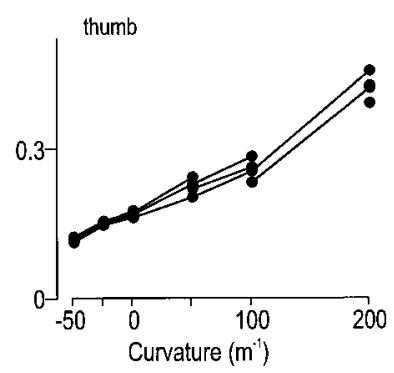

B
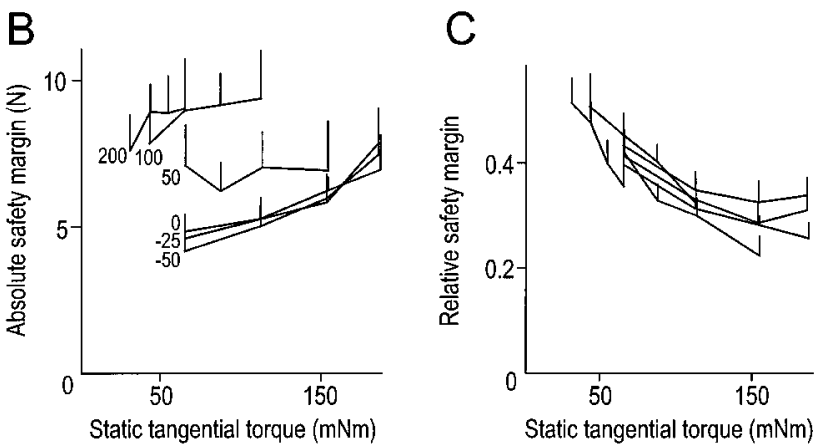

forces related to the movements. Indeed, this coordination appears to be an integral part of the process of planning arm movements and is also expressed under other forms of load, e.g., viscous, elastic, and composite loads (Flanagan and Wing, 1997).

The present study reveals that the sensorimotor programs controlling manipulative actions account not only for linear loads as described previously but also for torsional loads. Apparently, these programs model the effect of the total load comprising linear forces and tangential torques. However, there were brief moments during which this coupling was violated. These occurred when the subject generated fast fluctuations in torque to produce smooth angular movements during rapid rotations of the object. Such decoupling was expected, because it is known that rapid self-paced changes in linear load forces are not paralleled by correspondingly rapid changes in grip force (Flanagan and Tresilian 1994).

\section{Parametric adjustment of the relationship between torque and grip force with changes in surface curvature}

The balance between the grip force and the tangential torque was influenced by the curvature of the grasped surfaces throughout the trial; the grip force at any given torque increased (parametrically) with increasing curvature, resulting in an adequate safety margin against rotational slips, regardless of curvature. The magnitude of this safety margin (generally, $\sim 30-40 \%$ of the grip force) is comparable to the safety margin against linear slips found in vertical lifting tasks. The safety margin is also comparable to that found against rotational slips by Kinoshita et al. (1997) during the hold phase of their "rotational slip and hold" single digit task with flat grasp surfaces. Thus, curvature influenced the ratio of grip force to torque in a manner similar to the influence of the friction at the digit-object interface (Kinoshita et 
al., 1997). Furthermore, the changes in coordination of grip force and tangential load with changes in the properties of the object also resemble those that take place when objects are lifted vertically, subjecting the digits principally to linear force loads; the adopted grip-to-load force ratio is adjusted to the frictional condition (Johansson and Westling, 1984) and the shape of the object (Jenmalm and Johansson, 1997), such that an adequate grip force safety margin against slips is achieved. Interestingly, in a previous study, the curvature of the grasped surfaces had only a small effect on the grip force in tasks in which the object was lifted vertically, inducing primarily linear force loads (Jenmalm et al., 1998). Despite the small changes in grip force, subjects still maintained an adequate safety margin against slips. This is explained by the fact that the curvature has only a small effect on grip forces required to prevent slips under linear loads, in contrast to the large effect during torsional loads demonstrated in the current study.

\section{Rotational friction}

The coefficients of rotational friction measured by us for the flat surfaces are consistent with those measured by Kinoshita et al. (1997). (Note that the rotational slip coefficient reported in Results corresponds to the inverse of the coefficient of rotational friction.) In general terms, the contact mechanics that account for friction with loads comprising tangential torques, linear forces, and combinations thereof, is poorly understood. This applies to soft artificial fingers (Buss et al., 1996; Howe and Cutkosky, 1996) and even more so to human fingertips (El-Shimi, 1977; Han et al., 1996). In the latter case, the distribution of normal and shear stress within the contact area is unknown, even when a digit contacts a flat surface. With curved surfaces, the frictional limits reflect additional factors related to the complex mechanical contact between the surfaces and the digits. Indeed, the coefficient of rotational friction was influenced by the surface curvature; it decreased with an increase in curvature. However, there was a pronounced variability across subjects with regard to the effect of curvature. This variability is probably attributable to differences in digit mechanics, including anatomical and biomechanical factors such as nonlinear anisotropic elastic compliance, sudomotor activity, and degree of greasiness and hydration of the skin (Jenmalm et al., 1998). Unfortunately, current models of human fingertip mechanics are not yet sophisticated enough to allow a synthesis of such data for comparison with ours (cf. Srinivasan and Dandekar, 1996).

The coefficients measured by us were similar for the index finger and the thumb when the flatter surfaces were used but were slightly greater for the index finger when the more curved surfaces were used. This is probably attributable to differences in the nature of the contact for the two digits, but how factors like contact area would affect the coefficient of friction is not clear. A more pronounced consequence of differing contact was the partitioning of torque between the two digits. There was a wide range of torque ratios for the two digits, and the digit with the greater torque was usually the critical digit from the point of view of motor control and grasp stability.

\section{Control mechanisms adapting force and torque output to the mass distribution and surface curvature of the object}

For individual subjects, the rise time of the tilting movement was nearly constant across all trials, although the mass distribution of the object, and thus the target torque, varied between trials. The temporal development of torque and the coupled development of grip force was thus scaled parametrically by the mass distribution of the object to generate an angular trajectory that was approximately constant. The intended angular position was reached in a smooth and apparently critically damped manner, with an angular velocity profile that was essentially unimodal (Figs. $2 A-D, 3 A$, $6 A$ ). This behavior indicates that the motor output during the tilt reflected predictive control in which both the mass distribution and the target angle of the object were taken into account. The angular velocity profile resembled the "continuous" (Brooks, 1984) or "bell-shaped" (Bizzi and Abend, 1983) velocity profiles frequently reported for programmed intended arm and hand movements toward a target. Furthermore, the generation of complex torque waveforms during rapid rotations to obtain a smooth tilting movement in our task indeed indicates that the CNS used a refined internal model of the entire task dynamics. In general terms, there is abundant evidence that the CNS utilizes internal models of relevant limb mechanics, environmental objects, and task properties to structure the motor commands appropriately before their execution (Johansson and Westling, 1988b; Ghez et al., 1991; Johansson and Cole, 1992; Lacquaniti, 1992; Johansson, 1996; Miall and Wolpert, 1996; Flanagan and Wing, 1997).

When the mass distribution of the object changed, the appropriate modification of the torque and grip force was present from the beginning of the tilt (Fig. $3 A$ ). This implies that subjects used information related to the mass distribution of the object from the onset of the tilting movement. This information must have been obtained at the beginning of each trial, because the mass distribution, and therefore the target torque, was always changed between trials. One obvious cue in this respect was object weight, which covaried with the mass distribution. However, we cannot exclude the use of small "probing tilts" in the hold phase before the tilting movement. It is also possible that visual cues obtained by observing the interchangeable mass of the object could have provided cues on the current mass distribution. It has been shown that in lifting tasks, visual cues related to the mass of the object are used for forward parametric control of the force output (Gordon et al., 1991, 1993).

Similarly, when the surface curvature was changed, the appropriate modification of the grip force was present from the beginning of the tilt (Fig. $6 A-E$ ). There was no effect on the grip force of the surface curvature used in the previous trial. Thus, the subjects must have acquired information about surface curvature before the tilting movement. The curvature could have been determined from cutaneous afferent information obtained during the initial contact with the surfaces (Goodwin et al., 1995), in a manner similar to the acquisition of frictional information by tactile input during initial contact with a grasped surface (Johansson and Westling, 1984, 1987). It is also possible that subjects used visual cues. In lifting tasks in which the shape of the object changed, visual geometric cues modulated the force coordination in a feed-forward manner (Jenmalm and Johansson, 1997).

\section{REFERENCES}

Bizzi E, Abend W (1983) Posture control of trajectory formation in single- and multi-joint arm movements. In: Motor control mechanisms in health and disease, Vol 39 (Desmedt RE, ed), pp 31-45. New York: Raven.

Brooks VB (1984) How are "move" and "hold" programs matched? In: Cerebellar functions (Bloedel JR, Dichgans J, Precht W, eds), pp 1-22. Heidelberg: Springer.

Burstedt MKO, Edin BB, Johansson RS (1997) Coordination of fingertip forces during human manipulation can emerge from independent neural networks controlling each engaged digit. Exp Brain Res 117:67-79. 
Buss M, Hashimoto H, Moore JB (1996) Dexterous hand grasping force optimization. IEEE Trans Robotics Autom 12:406-418.

Cadoret G, Smith AM (1996) Friction, not texture, dictates grip forces used during object manipulation. J Neurophysiol 75:1963-1969.

El-Shimi AF (1977) In vivo skin friction measurements. J Soc Cosmet Chem 28:37-51.

Flanagan JR, Tresilian JR (1994) Grip load force coupling: a general control strategy for transporting objects. J Exp Psychol Hum Percept Perform 20:944-957.

Flanagan JR, Wing AM (1993) Modulation of grip force with load force during point-to-point arm movements. Exp Brain Res 95:131-143.

Flanagan JR, Wing AM (1995) The stability of precision grip forces during cyclic arm movements with a hand-held load. Exp Brain Res 105:455-464.

Flanagan JR, Wing AM (1997) The role of internal models in motion planning and control: evidence from grip force adjustments during movements of hand-held loads. J Neurosci 17:1519-1528.

Ghez C, Hening W, Gordon J (1991) Organization of voluntary movement. Curr Opin Neurobiol 1:664-671.

Goodwin AW, Browning AS, Wheat HE (1995) Representation of curved surfaces in responses of mechanoreceptive afferent fibers innervating the monkey's fingerpad. J Neurosci 15:798-810.

Gordon AM, Forssberg H, Johansson RS, Westling G (1991) Integration of sensory information during the programming of precision grip: comments on the contributions of size cues. Exp Brain Res 85:226-229.

Gordon AM, Westling G, Cole KJ, Johansson RS (1993) Memory representations underlying motor commands used during manipulation of common and novel objects. J Neurophysiol 69:1789-1796.

Han H, Shimada A, Kawamura S (1996) Analysis of friction on human fingers and design of artificial fingers. IEEE Int Conf Robotics Autom 4:3061-3066.

Howe RD, Cutkosky MR (1996) Practical force-motion models for sliding manipulation. Int J Robotics Res 15:557-572.

Jenmalm P, Johansson RS (1997) Visual and somatosensory information about object shape control manipulative fingertip forces. J Neurosci 17:4486-4499.
Jenmalm P, Goodwin AW, Johansson RS (1998) Control of grasp stability when humans lift objects with different surface curvatures. J Neurophysiol 79:1643-1652.

Johansson RS (1996) Sensory control of dexterous manipulation in humans. In: Hand and brain: the neurophysiology and psychology of hand movements (Wing AM, Haggard P, Flanagan JR, eds), pp 381-414. San Diego: Academic.

Johansson RS, Cole KJ (1992) Sensory-motor coordination during grasping and manipulative actions. Curr Opin Neurobiol 2:815-823.

Johansson RS, Westling G (1984) Roles of glabrous skin receptors and sensorimotor memory in automatic control of precision grip when lifting rougher or more slippery objects. Exp Brain Res 56:550-564.

Johansson RS, Westling G (1987) Signals in tactile afferents from the fingers eliciting adaptive motor responses during precision grip. Exp Brain Res 66:141-154.

Johansson RS, Westling G (1988a) Coordinated isometric muscle commands adequately and erroneously programmed for the weight during lifting task with precision grip. Exp Brain Res 71:59-71.

Johansson RS, Westling G (1988b) Programmed and triggered actions to rapid load changes during precision grip. Exp Brain Res 71:72-86.

Kinoshita H, Kawai S, Ikuta K, Teraoka T (1996) Individual finger forces acting on a grasped object during shaking actions. Ergonomics 39:243-256.

Kinoshita H, Bäckström L, Flanagan JR, Johansson RS (1997) Tangential torque effects on the control of grip forces when holding objects with a precision grip. J Neurophysiol 78:1619-1630.

Lacquaniti F (1992) Automatic control of limb movement and posture. Curr Opin Neurobiol 2:807-814.

Miall RC, Wolpert DM (1996) Forward models for physiological motor control. Neural Networks 9:1265-1279.

Srinivasan MA, Dandekar K (1996) Investigation of the mechanics of tactile sense using two-dimensional models of the primate fingertip. J Biomech Eng 118:48-55.

Westling G, Johansson RS (1984) Factors influencing the force control during precision grip. Exp Brain Res 53:277-284. 